communication from the FDA, they won't get the 'all hands on deck' approach that is promised by the breakthrough designation. For breakthrough therapies, senior FDA managers and reviewers are expected to work closely with drug sponsors to design collaborative, multidisciplinary development plans that hasten timelines to approval and minimize the number of patients exposed to less efficacious treatments or placebos.

"This is really meant to signal from the agency that if you have a drug that shows a really unprecedented activity early on, they want to work with you to find the best course forward, rather than have you go it alone," says Jeff Allen, executive director of
Friends of Cancer Research, a think tank and advocacy organization based in Washington, DC, that has actively championed the new breakthrough pathway.

Ultimately, Ivy expects patients to benefit as much as, if not more than, drug sponsors. Currently, individuals who are desperately seeking unapproved treatments but cannot enroll in a clinical trial must petition the FDA for a 'special exception use', yet drug companies are not always so keen to provide investigational medicines outside the confines of a controlled study. The breakthrough designation should increase drug access earlier on. "In the end," Ivy says, "I think the driver, goal and motivation for breakthrough therapies is to make treatments more widely accessible to patients who don't have other options."

However, onlookers say they hope the influence of a breakthrough designation won't be weakened by the FDA assigning it too frequently. "Like fast-track designations, I suspect they're going to give too many," says Greg Dombal, chief operating officer of Halloran Consulting Group, a Boston area firm that specializes in the life sciences industry. "And, in practice, its value could be slightly watered down because there are going to be a lot of things in there that shouldn't have that designation."

Elie Dolgin

\title{
Diagnostic lens turns to difficult-to-detect ovarian cancer
}

They say what you don't know can't hurt you, but that statement is particularly untrue when it comes to ovarian cancer. The most prevalent and aggressive type of ovarian cancer, known as high-grade serous carcinoma of the ovary (HGSC), is frequently diagnosed at stage 3 or 4 , when treatment success plummets. Early phases of the illness have few symptoms, and even the signs seen in later-stage disease-such as bloating, pelvic pain and urinary urgency - are not unique to cancer and can be misinterpreted.

Whereas screening procedures for prostate and breast cancer have become routine elements of doctor visits, ovarian cancer screening tests remain bogged down in the development stage. But recent findings offer hope: a pilot study led by scientists at the Johns Hopkins Kimmel Cancer Center in Baltimore published on 9 January showed that it is possible to detect cancerous mutations in DNA shed from ovarian and endometrial tumors that has made their way to the cervix can be detected using a liquid Papanicolaou (Pap) smear sample (Sci. Transl. Med. 5, 167ra4, 2013).

There is still a way to go, however. In the pilot study, only 10 of the 24 stage 1 cancers were detected. But the scientists behind the work are hopeful: "Our false positive rate was zero, which is exciting," says Luis Diaz, a Hopkins oncologist who led the study. "We hope to reproduce these results in a follow-up study with a larger number of cases with ovarian and endometrial cancer and in samples from healthy controls," Diaz says.

Others are working on molecular tests to glean more diagnostic information about ovarian health from liquid Pap smears, too. Alabama-based Swift Biotech has partnered with researchers at the University of South Alabama Mitchell Cancer Institute in Mobile who have been working on a proteomic-based test for the last five years. The analysis looks for a range of telltale proteins that originated in or near cancers in the ovary. "There is emerging molecular data that the majority of ovarian cancers originate in the fallopian tube," notes Rodney Rocconi, of the Mitchell Cancer Institute, one of the test's developers. A prospective validation trial, funded by a grant from the US National Institutes of Health, is currently ongoing in women with a detectable pelvic mass. Details about the test will be presented at the 2013 Society of Gynecologic Oncology annual meeting in Los Angeles this March.
Proponents of the liquid Pap test say that it provides superior detection of the local ovarian environment, but those developing blood tests for ovarian tumors say their assays have advantages such as convenience. And, in fact, a bloodbased test already exists for women who have a detected pelvic mass that could be cancer. In September 2011, the US Food and Drug Administration granted market approval through the 501 (k) pathway for a product from Pennsylvania-based Fujirebio Diagnostics that analyzes blood levels of the proteins HE4 and CA-125 with the so-called 'risk of ovarian malignancy algorithm'.

Measuring blood levels of CA-125 alone, without simultaneously measuring HE4, has shown limitations, however. A 2011 trial of over 78,000 women showed no benefit from a blood-based CA-125 biomarker and ultrasound combination test (JAMA 22, 2295-2303, 2011). The test led to frequent falsepositive results and too many unnecessary interventions and surgeries. And this past December, the US Preventive Services Task Force published a piece reaffirming its 2004 guidelines that recommended against screening for women who show no symptoms and who do not carry genetic mutations that increase ovarian cancer risk, such as the BRCA variants (Ann. Intern. Med. 157, 900-904, 2012).

But other companies say they have better blood biomarkers. Canada's Soricimed Biopharma is working on a new blood-based test to detect mRNA transcripts of the TRPV6 protein specific to ovarian and other epithelial cancers. It is also developing a magnetic resonance imaging-based imaging test in conjunction with a fluorescent drug that specifically binds a protein found on the surface of ovarian tumors as a screening diagnostic.

Experts in the field are optimistic, particularly about the assays that look at DNA. "I would not be surprised if a genomic test such as [the one being developed at Hopkins] would ultimately become commonplace, particularly for women at high risk for ovarian cancer," says Robert Soslow, director of gynecologic pathology at the Memorial Sloan-Kettering Cancer Center in New York. And, according to Soslow, the focus of any screening test for ovarian cancer should be detection of HGSC: "That is the home run."

Anna Azvolinsky 